IgA and IgG anti-TTG to increase sensitivity. There is a huge variation in the normal range of IgA TTG with the upper normal limit varying from 2.9 to 19 .

The survey results were presented (AH) at the annual regional network meeting generating an active discussion amongst the attending immunologists, paediatricians, dieticians and specialist nurses, with these conclusions.

- The huge variation in normal levels, with lack of standardisation in TTG testing, is an issue in the region, as highlighted nationally.

- The Immunologists highlighted variations even within the same serological test, when using different batches requiring labs to conduct regular quality control checks.

- A network consensus followed with regards to provision of dietary review for all patients (particularly $<10 \mathrm{X}$ serologic levels), to ensure adequate gluten intake before consideration of biopsy.

Conclusions $\mathrm{pCD}$ is a lifelong condition requiring an accurate diagnosis. The clinicians and immunologists continued to be challenged despite following the correct pathways and guidance. This study highlighting the strengths and benefits of a joint regional collaborative approach to help overcome some of these difficulties.

\section{G42 LONG TERM SAFETY AND EFFICACY OF SINGLE DOSE PARENTERAL IRON IN CHILDREN WITH INFLAMMATORY BOWEL DISEASE IN A LARGE TERTIARY CENTRE}

${ }^{1} \mathrm{C}$ Toh, ${ }^{2} \mathrm{R}$ Hubbard, ${ }^{2} \mathrm{P}$ Rao. ${ }^{1}$ Faculty of Medicine, Dentistry and Health, University of Sheffield, Sheffield, UK; ${ }^{2}$ Department of Gastroenterology, Sheffield Children's Hospital, Sheffield, UK

\subsection{6/archdischild-2018-rcpch.40}

Background Iron-deficiency anaemia is a common complication amongst children with inflammatory bowel disease (IBD). Although single-dose parenteral iron (PIN) preparations are an easily available treatment for children, there are still concerns surrounding its adverse reactions.

Aims The primary aim of this study was to evaluate the safety, side effects and efficacy of IV iron maltoside 1000 (Monofer) at 6 weeks, 3 months, 6 months and 1 year after treatment in children with IBD. Also, to look for any evidence of iron overload.

Methods A comprehensive search was performed using the hospital's IBD database to identify patients who have been given PIN from 2012 to 2016. Primary indication, underlying diagnosis, dose of iron, adverse reactions and laboratory values were among the parameters recorded. Dose calculations were based on the Ganzoni formula. PIN was used only if oral iron therapy is ineffective, not tolerated, not advisable or iron-deficiency anaemia with haemoglobin levels of $<100 \mathrm{~g} / \mathrm{L}$. Repeated measures ANOVA was conducted for statistical analysis.

Results A total of 29 patients were identified. The median age was 14 and median weight was $33.4 \mathrm{~kg}$. Two patients did not have the full data set. Repeated-measures ANOVA conducted on 27 patients showed that mean haemoglobin differed significantly between time points [F $(4,104)=29.416, \mathrm{p}<0.001]$. Residuals were approximately normally distributed. Post-hoc tests using the Bonferroni correction revealed that mean haemoglobin increased significantly by 6 weeks and remained stable thereafter $(\mathrm{p}<0.001)$.

Only one patient had an acute type 1 allergic reactions (not anaphylaxis). Two patients had hair loss at 3 months postinfusion which were unlikely to be secondary to iron overload. None of the patients had dysmetabolic iron overload syndrome (DIOS). All children had normal LFTs and GGTs with no evidence of diabetes, chronic fatigue or hepatosplenomegaly in their follow up.

Conclusion PIN appears to have sustained efficacy in the treatment of iron deficiency anaemia in children with IBD. Iron status increased significantly at 6 weeks and sustained till 1 year post-infusion. The immediate reaction rate was $3.7 \%$ and none of the remaining patients had any side effects including any evidence of DIOS.

\section{G43(P) DIFFICULTIES IN COMPLYING WITH THE BSPGHAN GUIDELINES FOR DIAGNOSIS AND MANAGEMENT OF CELIAC DISEASE (2013)}

${ }^{1} \mathrm{C}$ Mitchell, ${ }^{1} \mathrm{~A}$ Thakur, ${ }^{1,2} \mathrm{~N}$ Onyeador, ${ }^{1}$ I Bilal. ${ }^{1}$ General Paediatrics, Ashford and St Peter's NHS Foundation Trust, London, UK; ${ }^{2}$ Paediatric Gastroenterology, St George's University NHS Foundation Trust, London, UK

\subsection{6/archdischild-2018-rcpch.41}

Aims To audit our paediatric department's practice of diagnosis and management of celiac disease, comparing it to the BSPGHAN and Coeliac UK guidance published in 2013, to review and consider improvements to our current practice. Method Collection of data from 2015-2017 including all patients aged $<18$ years old with a positive tissue transglutaminase (tTG). Electronic records were used to gather information regarding other serological tests (endomysial antibodies (EMA)), genetic tests (HLA DQ2/DQ8), and biopsy results; as well as time-to-follow up with both the dietician and clinician. Results Of the 55 children with a positive tTG there were sufficient data to include 40. 10/40 were asymptomatic; 30/40 symptomatic, and the ratio of females to males was $3.4: 1$.

This audit demonstrated that $67.5 \%$ were diagnosed according to the national guidelines, and for those cases where the guidance was not adhered to it was most commonly because a biopsy had not been performed (see table 1 below).

\begin{tabular}{lc} 
Abstract G43(P) Table 1 & 2 \\
\hline No genetic investigations & 1 \\
\hline No genetic investigations or inadequate serology & 2 \\
Inadequate serology and no biopsy where & \\
indicated & 6 \\
No biopsy performed where indicated & 2 \\
Investigated on a gluten-free diet & \\
\hline
\end{tabular}

Only $17 \%$ of patients were seen by the dietician within 2 weeks following diagnosis, and only 40\% within 6 months. $77 \%$ were seen by a clinician at 12 months but only $47 \%$ had a repeat tTG at this appointment. Finally, only $20 \%$ of those eligible were transitioned to adult services.

Conclusion Clinicians face many difficulties when diagnosing celiac disease. Most notably convincing families to return to a gluten-containing diet prior to further investigations; and 